\title{
The evaluation of cartilage differentiations using transforming growth factor beta3 alone and with combination of bone morphogenetic protein- 6 on adult stem cells
}

\begin{abstract}
In our quest to standardize our formula for a clinical trial, transforming growth factor-beta3 (TGF- $\beta 3$ ) alone and in combination with bone morphogenetic protein-6 (BMP-6) were evaluated for their effectiveness in cartilage differentiation. Bone Marrow Stem Cells (BMSCs) and Adipose Derived Stem Cells (ADSCs) were induced to chondrogenic lineage using two different media. Native chondrocytes served as positive control. ADSCs and BMSCs proved multipotency by tri-lineage differentiations. ADSC has significantly higher growth kinetics compare to Chondrocyte only $\mathrm{p} \leq 0.05$. Using TGF- $\beta 3$ alone, BMSC revealed higher expressions for hyaline cartilage genes compare to ADSCs. Chondrocyte has significantly higher early chondrogenic markers expression to ADSCs and BMSCs, while BMSCs was only higher to ADSC at chondroadherin, $\mathrm{p} \leq 0.0001$. On mature chondrogenic markers, chondrocytes were significantly higher to ADSCs and BMSCs for aggrecan, collagen IX, sry (sex determining region y)-box9, collagen II and fibromodullin; and only to ADSC for collagen XI. BMSC was higher to ADSC for aggrecan and collagen IX, $\mathrm{p} \leq$ 0.0001 . The combination of TGF- $\beta 3$ + BMP- 6 revealed increased gene expressions on both BMSCs and ADSCs for early and mature chondrogenic markers, but no significance difference. For dedifferentiation markers, ADSC was significantly higher to chondrocyte for collagen I. Glycosaminoglycan evaluations with both formulas revealed that chondrocytes were significantly higher to ADSCs and BMSCs, but none was significant to each other, $\mathrm{p} \leq$ 0.0001. Combination of $10 \mathrm{ng}$ TGF- $\beta 3$ with $10 \mathrm{ng}$ of BMP-6 enhanced chondrogenic potentials of BMSCs and ADSCs compare to TGF- $\beta 3$ alone. This could be the ideal cocktail for either cell's chondrogenic induction.
\end{abstract}

Keyword: Cartilage; Cell therapy; Chondrogenic induction; Stem cells 